\title{
Contribuição de quintais agroflorestais na conservação de plantas da Caatinga, Município de Caruaru, PE, Brasil ${ }^{1}$
}

\author{
Alissandra Trajano Nunes Florentino², Elcida de Lima Araújo² e Ulysses Paulino de Albuquerque ${ }^{2,3}$
}

Recebido em 16/03/2006. Aceito em 1/08/2006

\begin{abstract}
RESUMO - (Contribuição de quintais agroflorestais na conservação de plantas da Caatinga, Município de Caruaru, PE, Brasil). A presente pesquisa foi realizada na zona rural do município de Caruaru, tendo como objetivo estudar a diversidade florística e a contribuição de quintais agroflorestais para a conservação da diversidade local. O trabalho foi conduzido a partir de entrevistas semi-estruturadas, associadas à técnica da turnê-guiada, realizada com os mantenedores dos quintais. Paralelamente, fez-se medição da área do quintal e de todos os indivíduos lenhosos com DNS (diâmetro ao nível do solo) $\geq 3 \mathrm{~cm}$. Registrou-se 25 quintais, nos quais foram relacionadas 84 espécies, pertencentes a 68 gêneros e 35 famílias, destacando-se as famílias Euphorbiaceae (10 spp.), Anacardiaceae ( 7 spp.), Caesalpiniaceae (6 spp.), Mimosaceae (6 spp.) e Myrtaceae (6 spp.). As espécies introduzidas (55 espécies) dominaram nos sistemas em relação às nativas. Apesar destas terem sido menos freqüentes nos quintais agroflorestais, elas são importantes para conservação da diversidade local devido, principalmente, ao uso madeireiro, o que poderia implicar na redução do impacto na vegetação local. Este dado é um indicativo de que por meio de um plano de manejo adequado, os quintais agroflorestais constituem uma alternativa, de baixo custo, para a conservação da diversidade local.
\end{abstract}

Palavras-chave: sistemas agroflorestais, etnobotânica, conservação da biodiversidade, florestas secas, plantas alimentícias, plantas medicinais

\begin{abstract}
Contribution of homegardens to the conservation of caatinga plants, Municipality of Caruaru, Pernambuco State, Brazil). The research presented here was undertaken in a rural area within the municipality of Caruaru. It was designed to evaluate the floristic diversity of homegardens and their role in conserving local diversity. Semi-structured interviews with the people responsible for these plots were carried out, in association with the technique of guided excursions. The sizes of the homegardens were measured, and all woody plants with a DGL (Diameter at Ground Level) $\geq 3 \mathrm{~cm}$ were recorded. A total of 25 plots were examined, containing 84 species of plants, belonging to 68 genera and 35 families. The most species rich families were Euphorbiaceae (10 species), Anacardiaceae (7), Caesalpiniaceae (6), Mimosaceae (6), and Myrtaceae (6). Introduced species (55 species) were most prevalent. Eventhough native species were less frequent than introduced species in these homegardens, these plots are important for the conservation of local diversity, principally because of the protection they provide to local woody species by reducing the impact of tree harvesting. This information indicates that, when properly managed, homegardens represent a low-cost alternative for conserving local diversity.
\end{abstract}

Key words: Agroforest systems, ethnobotany, biodiversity conservation, dry forests, edible plants, medicinal plants

\section{Introdução}

A Caatinga é uma vegetação seca, característica da região Nordeste do Brasil, destacando-se por apresentar uma grande variedade de fisionomias e uma boa diversidade de espécies endêmicas (MMA 2002). A região é caracterizada por possuir baixos níveis de pluviosidade, variando entre um mínimo de $250 \mathrm{~mm}$ e máximo 800 mm em áreas intermediárias (MMA 2002; Maia 2004). A área vem sofrendo impacto desde tempos muito remotos, com a entrada dos colonizadores no Brasil em busca de áreas para criação de gado (Andrade-Lima 1981; Diegues \& Arruda 2001). Desde então, a Caatinga é utilizada para atividades agropecuárias, sendo este um dos fatores que contribuem para a devastação local (Andrade-Lima 1981; Sampaio 2002).

Apesar das adversidades e das severas condições ambientais, a população local adaptou-se a tais condições, sobrevivendo por meio do cultivo de plantas como o feijão (Phaseolus vulgaris L.), o milho (Zea mays L.) e a macaxeira (Manihot sp.) para complementação alimentar em áreas específicas para monocultura ou em quintais (Andrade-Lima 1981;

\footnotetext{
1 Parte da dissertação de Mestrado da primeira Autora

2 Universidade Federal Rural de Pernambuco, Departamento de Biologia, Área de Botânica, Programa de Pós-graduação em Botânica, Rua Dom Manoel de Medeiros s/n, Dois Irmãos, 52171-900 Recife, PE, Brasil (www.ufrpe.br/lea)

3 Autor para correspondência: upa@db.ufrpe.br
} 
Sampaio 2002). Esses quintais podem ser compreendidos como parte integrante de sistemas agroflorestais. Os sistemas agroflorestais envolvem o cultivo de plantas lenhosas, associado à prática de monocultura e à criação de animais domésticos, em uma determinada área (Fernandes \& Nair 1996).

Os quintais agroflorestais representam uma unidade agrícola de uso tradicional do solo, considerados como uma das formas mais antigas de uso da terra, promovendo a sustentabilidade para milhões de pessoas no mundo (Nair 1986). Sua principal finalidade é a produção de alimento para complementação da dieta familiar e as práticas de manejo são consideradas ecologicamente sustentáveis (Nair 1986; 1993a; b; 2001; 2004; Rico-Gray et al. 1990; Torquebiau 1992; Blanckaert et al. 2002). Além do potencial de sustentabilidade ecológica, os quintais são considerados sistemas alternativos de complementação da demanda alimentar (Soewarwoto et al. 1985). Uma alta diversidade de espécies, com múltiplas finalidades, é cultivada nos quintais, tais como plantas usadas para construção, combustível, artesanato, ornamental, sombra, fibra, religião e medicina (Fernandes \& Nair 1986; Beatriz et al. 1998; Albuquerque et al. 2005; Blanckaert et al. 2002; Wezel \& Bender 2003).

É evidente a importância dos quintais sob vários aspectos, mas pouca atenção ainda tem sido dada a essas práticas, especialmente no Brasil. Para se ter uma idéia, só na Ásia até a década de 90, os quintais foram objeto de estudo em aproximadamente $40 \%$ das publicações, sendo a maioria de cunho qualitativo, descrevendo o ambiente, sua composição florística e função. Até 2004, somam-se mais de 83 publicações no Sul e Sudeste daquele país (Nair 2004). Além da Ásia, destacam-se América Central e Oeste da África, com grandes índices de publicações sobre quintais (Nair 2004).

No Brasil, há uma considerável quantidade de estudos florísticos em quintais, a maioria apresentando dados qualitativos com descrições da estrutura, composição, organização e manutenção dessas práticas (Anderson et al. 1985; Emperaire \& Pinton 1986; Lamont et al. 1999). Na região Norte as pesquisas são consideradas avançadas (Padoch \& Jong 1991; Lamont et al. 1999; Santos et al. 2004). Em ambiente semi-árido, os trabalhos são praticamente escassos, com apenas dois estudos que evidenciaram uma expressiva riqueza e diversidade de espécies (cf. Emperaire \& Pinton 1986; Albuquerque et al. 2005). Contudo, as pesquisas na área são consideradas deficientes ou escassas, havendo muito a se explorar do ponto de vista florístico, estrutural, ecológico, sócioeconômico e cultural.

Com base nessas considerações, o presente estudo visa contribuir para o conhecimento da estrutura e florística de quintais agroflorestais de uma área de caatinga, no município de Caruaru, Pernambuco (Nordeste do Brasil), a fim de responder aos seguintes questionamentos: Os quintais são floristicamente diversificados? Quais são as plantas lenhosas nativas mantidas nessas áreas? Essas áreas podem contribuir para a conservação da biodiversidade local? Que relações existem entre a riqueza do quintal com o seu tamanho, idade, função e idade do mantenedor? Quem é o principal responsável pela manutenção do quintal? Que técnicas de manejo são utilizadas e qual a principal fonte de obtenção das espécies nos quintais?

\section{Material e métodos}

Área de estudo - O trabalho de campo foi realizado no município de Caruaru, Agreste Pernambucano, Nordeste do Brasil. Caruaru possui uma área de $928 \mathrm{~km}^{2}$ (8 $144^{\prime} 19^{\prime \prime} \mathrm{S}$ e $\left.35^{\circ} 55^{\prime} 17^{\prime \prime} \mathrm{W}\right)$, com $550 \mathrm{~m}$ de altitude, e dista $136 \mathrm{~km}$ da capital do Estado de Pernambuco, de clima semi-árido quente com temperatura média anual em torno de $24{ }^{\circ} \mathrm{C}$ (FIDEM 2003). A precipitação anual é cerca de 609 mm, com chuvas concentradas nos meses de junho e julho, mas em períodos atípicos atinge pouco mais que $1.000 \mathrm{~mm}$ (Empresa Pernambucana de Pesquisa Agropecuária: IPA 2004). Possui uma população de 253.634 habitantes e densidade demográfica de $273,3 \mathrm{hab} / \mathrm{km}^{2}$ (FIDEM 2003). A cidade é reconhecida nacionalmente como o maior centro econômico do Nordeste devido à "Feira de Caruaru", onde são comercializados vários produtos, desde artesanatos a alimentos, constituindo a base da economia local. Além disso, a agricultura e a pecuária são também importantes para a economia das comunidades rurais (Caruaru: o portal - 2004).

A comunidade de Riachão de Malhada de Pedra, localizada nas vizinhanças do fragmento de caatinga pertencente ao IPA, possui 117 unidades familiares e 438 habitantes (Programa de Saúde da Família 2004). Uma das principais fontes de renda da população local é a agricultura, mas muitos trabalham como autônomos no comércio local, na produção de roupas ou em fazendas da região. As casas são de alvenaria, não há calçamento nem saneamento básico; a energia elétrica é fornecida a todas as residências, mesmo as mais afastadas, e o abastecimento de água é feito uma vez 
por semana, através de um chafariz municipal com sede no povoado de Malhada de Pedra (R.F.P. Lucena, comunicação pessoal, 2006). Na maioria das casas existem reservatórios de água, naturais ou artificiais. A estrutura da comunidade é simples: na parte central há telefone público, bares e um templo da Igreja católica. Os cuidados médicos ficam por conta de dois postos de saúde, localizados próximo à comunidade, e por meio do programa de saúde da família. Em casos de enfermidades mais graves as pessoas se deslocam para Caruaru ou para cidades vizinhas. Quanto à educação, é oferecida instrução escolar até o nível fundamental. A partir do nível médio, os alunos se deslocam até a comunidade de Serra Velha (R.F.P. Lucena, comunicação pessoal, 2006). Mais informações sobre a área de trabalho, e diferentes aspectos do uso dos recursos vegetais, podem ser vistas em Albuquerque et al. (2006), Lucena et al. (2007) e Monteiro et al. (2006a,b).

Métodos - O trabalho de campo foi realizado no período de março/2004 a julho/2005 nas residências que possuíam quintais agroflorestais típicos (mantidos com o trabalho familiar e tecnologia tradicional) e ativos. Um total de 25 quintais foi contabilizado, para toda a comunidade e, após a seleção dos mesmos e com consentimento prévio do mantenedor, em cada quintal estimou-se a área total. Paralelamente, para todos os indivíduos presentes na área (exceto aqueles que compunham as cercas que delimitavam os quintais) foram tomadas medidas de altura e diâmetro ao nível do solo (DNS) $\geq 3 \mathrm{~cm}$ (Albuquerque et al. 2005). O material botânico coletado foi etiquetado no local com o número e a data de coleta, acondicionado em saco plástico e processado ainda em campo. Em seguida, foi encaminhado para o Laboratório de Etnobotânica Aplicada, da Universidade Federal Rural de Pernambuco. Aidentificação do material foi feita com auxílio da literatura e/ou confirmada por especialistas da área, sendo o material testemunho depositado no Herbário Professor Vasconcelos Sobrinho-PEUFR, da Universidade Federal Rural de Pernambuco.

A fisionomia dos quintais foi descrita por um diagrama de perfil, realizado com base em dois componentes: vertical e horizontal, de acordo com a localização das espécies (Millat-e-Mustafa 1998). A estrutura vertical foi traçada com base nas medidas de DNS e altura das espécies lenhosas da área. A estrutura horizontal da vegetação foi avaliada por meio da localização das espécies dentro do quintal (Millate-Mustafa 1998). A relação entre a estratificação do quintal e as formas de uso foram abordadas com base nessas análises. Os cálculos de abundância e freqüência foram realizados de acordo com Araújo \& Ferraz (2004). Esses dados foram também utilizados para um esboço de classificação "a posteriori” dos quintais, associado aos dados de aproveitamento local das plantas.

Entrevistou-se um total de 25 pessoas responsáveis pela manutenção das práticas em suas respectivas propriedades. A amostra compreendeu 17 mulheres e oito homens com idade variando entre $20 \mathrm{e}$ 80 anos. As informações sobre as plantas foram obtidas por meio de observação direta e entrevistas semiestruturadas, com auxílio de um gravador portátil (com o consentimento de cada informante) e um diário de campo para informações adicionais (Albuquerque \& Lucena 2004). Concomitantemente, utilizou-se a técnica da turnê-guiada, na qual o mantenedor foi convidado a fazer uma caminhada pelo quintal durante a entrevista, fornecendo informações específicas sobre as plantas presentes (Albuquerque \& Lucena 2004). As plantas citadas foram enquadradas nas seguintes categorias de uso: alimentar, forragem, medicinal, ornamental, madeireira (incluindo os usos para construção, tecnologia e combustível), produção de sombra e outros usos (correspondendo a uma miscelânea de finalidades citadas ocasionalmente, como mágico-religioso e fibra). Tais categorias representam uma junção de aspectos éticos e êmicos.

Os questionamentos envolveram perguntas de caráter sócio-econômico, tais como: idade, tempo de moradia, sexo, principal ocupação, número de pessoas habitando a casa, principal fonte de renda e perguntas sobre as técnicas de manejo dos quintais (Millat \& Mustafa 1998): 1. Usa algum material no manejo das plantas? 2. Se usa, qual seria? 3. Segue algum critério para selecionar as plantas a serem cultivadas? 4 . Existe alguma área preferencial do quintal para o cultivo de determinadas plantas? 5. Qual o procedimento para com as plantas que crescem espontaneamente? 6. Qual a pessoa da residência responsável pela manutenção do quintal?

Análise dos dados - Após a análise da riqueza dos quintais fez-se associações com o tamanho, função, tempo de implantação do quintal e, ainda, idade do mantenedor, utilizando o coeficiente de correlação de Spearman (Sokal \& Rolf 1995). A contribuição dos quintais na conservação da diversidade foi avaliada por meio do número de espécies lenhosas nativas presentes nos quintais em relação ao fragmento de 
mata vizinho a comunidade (a partir dos dados obtidos em Lucena et al. 2007), usando o teste de Quiquadrado $\left(\chi^{2}\right)$. O mesmo teste foi usado para analisar a proporção entre riqueza e abundância de plantas exóticas e nativas. O teste G de Williams (Sokal \& Rolf 1995) foi usado para comparar a proporção de plantas exóticas e nativas por categorias de uso. Todas as análises foram realizadas empregando-se o software Bioestat 2.0 (Ayres et al. 2000).

\section{Resultados e discussão}

Florística - A composição florística e a distribuição das espécies nos quintais são determinadas por fatores externos e internos, como função e tamanho do quintal, bem como fatores socio-econômicos e culturais, além da influência direta da família que seleciona as espécies de acordo com as suas necessidades (Nair 1986). Nos quintais de Riachão de Malhada de Pedra, registrou-se 84 espécies, pertencentes a 68 gêneros e 35 famílias (Tab. 1). As famílias mais representativas em relação a diversidade foram Euphorbiaceae (10 spp.), Anacardiaceae (7 spp.), Caesalpiniaceae (6 spp.), Mimosaceae (6 spp.) e Myrtaceae (6 spp.), com várias espécies comuns aos quintais tropicais, como Musa paradisiaca, Cocos nucifera, Psidium guajava, Mangifera indica, Carica papaya, Citrus sp. (Rico-Gray et al. 1985; 1990; Clerck \& Negrero-Castillo 2000; Wezel \& Bender 2003; Kehlenbeck \& Mass 2004; Albuquerque et al. 2005).

Registrou-se uma alta riqueza e abundância de espécies, contabilizando-se 1.461 indivíduos nos 25 quintais amostrados, quando comparado com estudos similares na Caatinga pernambucana (Albuquerque et al. 2005). Deste universo, 1.036 indivíduos correspondem às plantas introduzidas, as quais totalizam 56 espécies, sendo mais freqüentes Spondias purpurea (seriguela), Psidium guajava (goiaba) e Malpighia glabra (acerola). Os 425 indivíduos restantes representam plantas nativas da caatinga, englobando 28 espécies.

É comum observar a presença de espécies nativas nos quintais agroflorestais das regiões tropicais úmidas e áridas, porém em todos há um domínio de plantas exóticas (Rico-Gray et al. 1990; Nair 2004; Albuquerque et al. 2005). Pesquisas apontam que em muitos quintais, a presença de plantas nativas associadas às espécies introduzidas torna estas áreas semelhantes a sistemas florestais naturais, aparentando um ambiente equilibrado ecologicamente, porém este ainda é um tema de discussão para os cientistas da área (Barrera 1980; Nair 2004; Wiersum 2004).

Nos quintais de Riachão de Malhada de Pedra, a proporção de espécies e indivíduos de plantas exóticas, em relação às plantas nativas, foi significativamente maior $\left(\chi^{2}=5,86 ; p<0,05\right)$. Das plantas nativas, $47,2 \%$ (17 espécies) ocorrem no fragmento adjacente à comunidade, sendo mais freqüentes e abundantes nos quintais Piptadenia stipulacea e Acacia paniculata. Todavia, isso não corresponde a uma parcela significativa da diversidade vegetal registrada localmente $\left(\chi^{2}=5,86 ; p>0,05\right)$.

As espécies nativas da área, cultivadas nos quintais, possuem uso madeireiro, destacando-se Piptadenia stipulacea e Acacia paniculata, destinadas à produção de lenha. Tal resultado, encontrado para Riachão, é um indicativo que os quintais estudados podem contribuir na conservação da biodiversidade, uma vez que o hábito de cultivar plantas nativas pode diminuir a pressão de uso sobre a vegetação local. Esperava-se, no entanto, encontrar uma maior diversidade de espécies nativas, devido à proximidade ao fragmento de caatinga, como visto por Albuquerque et al. (2005) nos quintais do Município de Alagoinha, semi-árido do Nordeste brasileiro.

No cômputo geral, a espécie mais freqüente foi Anacardium occidentale, com 80 indivíduos presentes em 20 quintais, seguida de S. purpurea, com 76 indivíduos em 19 quintais, M. glabra e P. guajava, com 68 indivíduos em 17 quintais. Todas essas espécies são frutíferas de grande importância na complementação dietética da unidade familiar.

De acordo com a forma de uso e associada à abundância e freqüência das espécies, os quintais podem ser classificados em três categorias: 1. Dominados por plantas frutíferas, constituindo as espécies mais importantes, como Musa paradisiaca, A. occidentale, M. glabra, S. purpurea, P. guajava, Spondias mombim, Citrus limonum e Cocos nucifera, com algumas utilizadas também como medicinais; 2. Dominados por plantas ornamentais e, ocasionalmente, com outros usos, destacando Hibiscus rosa-sinensis, Jatropha mollissima e Jatropha gossypiifolia; e 3. Dominados por plantas de usos múltiplos, com baixa freqüência e abundância, como Senna martiana e Leucaena leucocephala.

Estrutura dos quintais - Encontrou-se uma expressiva relação do tamanho dos quintais com a riqueza $(\mathrm{r}=0,69 ; \mathrm{P}=0,001)$ e a abundância $(\mathrm{r}=0,60$; $\mathrm{P}=0,0001)$. Para Lamont et al. (1999) há uma relação direta entre o tamanho e a riqueza, embora outros estudos não apoiem totalmente esta assertiva (cf. 
Tabela 1. Espécies presentes nos quintais de Riachão de Malhada de Pedra, no município de Caruaru, Pernambuco (Nordeste do Brasil). Categorias de uso: 1 = alimentar; 2 = forragem; 3 = medicinal; $4=$ ornamental; $5=$ madeireira; $6=$ produção de sombra; $7=$ Outros usos. Ab. $=$ Abundância. Oc. $=$ Ocorrência. Fr. $=$ Freqüência relativa. Status: $\mathrm{N}=$ Nativa; $\mathrm{E}=$ exótica.

\begin{tabular}{|c|c|c|c|c|c|c|}
\hline Família/Espécie & Nome vulgar & Oc. & $\mathrm{Ab}$. & Fr. & Status & Usos \\
\hline \multicolumn{7}{|l|}{ AMARANTHACEAE } \\
\hline Celosia cristata L. & crista-de-galo & 1 & 4 & 0,24 & $\mathrm{E}$ & 4 \\
\hline \multicolumn{7}{|l|}{ ANACARDIACEAE } \\
\hline Anacardium occidentale L. & caju & 20 & 80 & 4,78 & $\mathrm{E}$ & 1,3 e 6 \\
\hline Mangifera indica $\mathrm{L}$. & mangueira & 10 & 40 & 2,39 & $\mathrm{E}$ & 1 e 6 \\
\hline Myracrodruon urundeuva (Engler) Fr. Allemão & aroeira & 1 & 4 & 0,24 & $\mathrm{~N}$ & 3 e 6 \\
\hline Schinopsis brasiliensis Engler & baraúna & 1 & 4 & 0,24 & $\mathrm{~N}$ & 6 \\
\hline Spondias purpurea L. & seriguela & 19 & 76 & 4,55 & $\mathrm{E}$ & 1 \\
\hline S. tuberosa Arr. Cam. & imbu & 3 & 12 & 0,72 & $\mathrm{~N}$ & 1 e 6 \\
\hline S. monbim L. & cajá & 13 & 52 & 3,11 & $\mathrm{E}$ & 1 e 6 \\
\hline \multicolumn{7}{|l|}{ ANNONACEAE } \\
\hline Annona muricata $\mathrm{L}$. & graviola & 2 & 8 & 0,48 & $\mathrm{E}$ & 1 \\
\hline Annona sp. & aticum & 1 & 4 & 0,24 & $\mathrm{E}$ & 1 \\
\hline A. squamosa L. & pinha & 13 & 52 & 3,11 & $\mathrm{E}$ & 1 e 6 \\
\hline \multicolumn{7}{|l|}{ APOCYNACEAE } \\
\hline Catharanthus roseus (L.) G. Don & boa-noite & 1 & 4 & 0,24 & $\mathrm{E}$ & 4 \\
\hline Nerium oleander L. & espirradeira & 7 & 28 & 1,67 & $\mathrm{E}$ & 4 \\
\hline \multicolumn{7}{|l|}{ ARECACEAE } \\
\hline Syagrus sp. & catolé & 3 & 12 & 0,72 & $\mathrm{~N}$ & 1 e 4 \\
\hline Cocos nucifera $\mathrm{L}$. & côco & 12 & 48 & 2,87 & $\mathrm{E}$ & 1,4 e 6 \\
\hline \multicolumn{7}{|l|}{ ASTERACEAE } \\
\hline Dahlia coccinea Cav. & rosa-branca e rosa & 3 & 12 & 0,72 & $\mathrm{E}$ & 4 \\
\hline Tithonia diversifolia A. Gray & girassol & 1 & 4 & 0,24 & $\mathrm{E}$ & 4 \\
\hline Vernonia condensata Toledo & alcachofra & 1 & 4 & 0,24 & $\mathrm{E}$ & 3 \\
\hline \multicolumn{7}{|l|}{ BIGNONIACEAE } \\
\hline Tecoma stans (L.) Juss ex Kunth & ipezinho & 2 & 8 & 0,48 & $\mathrm{E}$ & 4 \\
\hline \multicolumn{7}{|l|}{ BORAGINACEAE } \\
\hline Cordia trichotoma (Vel.) Arráb. ex Steud. & frei-jorge & 3 & 12 & 0,72 & $\mathrm{~N}$ & 5 \\
\hline \multicolumn{7}{|l|}{ BURSERACEAE } \\
\hline Comiphora leptophloeos (Mart.) J.B. Gillett & amburana & 3 & 12 & 0,72 & $\mathrm{~N}$ & 6 \\
\hline \multicolumn{7}{|l|}{ CAESALPINIACEAE } \\
\hline Bauhinia cheilantha (Bong.) Steud. & mororó & 2 & 8 & 0,48 & $\mathrm{~N}$ & 3 e 4 \\
\hline Caesalpinia pulcherrima L. & flambloyanzinho & 1 & 4 & 0,24 & $\mathrm{E}$ & 4 \\
\hline Senna martiana (Benth.) Irwin \& Barneby & canafístula & 2 & 3 & 0,18 & $\mathrm{~N}$ & 2,4 e 6 \\
\hline S. splendida (Vog.) Irwin \& Barneby & canafístula & 1 & 4 & 0,24 & $\mathrm{~N}$ & 4 e 6 \\
\hline $\begin{array}{l}\text { S. spectabilis var. excelsa (Schrad.) H.S. Irwin } \\
\quad \& \text { Barneby }\end{array}$ & canafístula & 1 & 12 & 0,72 & $\mathrm{~N}$ & 4 e 6 \\
\hline Tamarindus indica $\mathrm{L}$. & tamarindo & 1 & 4 & 0,24 & $\mathrm{E}$ & 1 e 6 \\
\hline \multicolumn{7}{|l|}{ CAPARIDACEAE } \\
\hline Crataeva tapia $\mathrm{L}$. & trapiá & 5 & 20 & 1,20 & $\mathrm{~N}$ & 1 e 6 \\
\hline Capparis hastata Jacq. & feijão-de-boi & 2 & 8 & 0,48 & $\mathrm{~N}$ & 5 \\
\hline \multicolumn{7}{|l|}{ EUPHORBIACEAE } \\
\hline Croton argyroglossum Baill. & velame-branco & 1 & 4 & 0,24 & $\mathrm{~N}$ & 5 \\
\hline C. rhamnifolius (Kunth.) Muell. Arg. & velame & 1 & 4 & 0,24 & $\mathrm{~N}$ & 5 \\
\hline Euphorbia cotinifolia L. & crote-rôxo & 2 & 8 & 0,48 & $\mathrm{E}$ & 4 \\
\hline Jatropha curcas L. & pinhão-manso & 3 & 12 & 0,72 & $\mathrm{~N}$ & 7 \\
\hline J. gossypiifolia $\mathrm{L}$. & pinhão-rôxo & 10 & 40 & 2,39 & $\mathrm{E}$ & 7 \\
\hline J. mollissima (Pohl) Baill. & pinhão-brabo & 11 & 44 & 2,63 & $\mathrm{~N}$ & 5 e 7 \\
\hline Sapium sp. & laco-benedita & 5 & 20 & 1,20 & $\mathrm{E}$ & 4 \\
\hline Ricinus communis L. & carrapateira & 2 & 8 & 0,48 & $\mathrm{E}$ & 7 \\
\hline Schefflera arboricola (Hayata) Merr & chefrera & 1 & 4 & 0,24 & $\mathrm{E}$ & 4 \\
\hline \multicolumn{7}{|l|}{ FABACEAE } \\
\hline Erythrina velutina Willd. & mulungu & 3 & 12 & 0,72 & $\mathrm{~N}$ & 4 e 6 \\
\hline \multicolumn{7}{|l|}{ GERANIACEAE } \\
\hline Geranium sp. & pinhãozinho & 1 & 4 & 0,24 & $\mathrm{E}$ & 4 \\
\hline
\end{tabular}


Tabela 1 (continuação)

\begin{tabular}{|c|c|c|c|c|c|c|}
\hline Família/Espécie & Nome vulgar & Oc. & $\mathrm{Ab}$. & Fr. & Status & Usos \\
\hline \multicolumn{7}{|l|}{ LAMIACEAE } \\
\hline Ocimum gratissimum $\mathrm{L}$. & alfavaca-louro & 3 & 12 & 0,72 & $\mathrm{E}$ & 3 \\
\hline Plectranthus sp. 1 & hortelã & 3 & 12 & 0,72 & $\mathrm{E}$ & 3 \\
\hline Plectranthus sp. 2 & hortelã da folha graúda & 1 & 4 & 0,24 & $\mathrm{E}$ & 3 \\
\hline \multicolumn{7}{|l|}{ LAURACEAE } \\
\hline Persea americana Mill. & abacate & 8 & 32 & 1,91 & $\mathrm{E}$ & 1 e 6 \\
\hline \multicolumn{7}{|l|}{ MALPIGHIACEAE } \\
\hline Malpighia glabra L. & acerola & 17 & 68 & 4,07 & $\mathrm{E}$ & 1 \\
\hline \multicolumn{7}{|l|}{ MALVACEAE } \\
\hline Gossypium hirsutum L. & algodão & 1 & 4 & 0,24 & $\mathrm{E}$ & 7 \\
\hline Hibiscus rosa-sinensis L. & papoula & 11 & 44 & 2,63 & $\mathrm{E}$ & 4 \\
\hline Abutilon striatum Dicks & balão & 1 & 4 & 0,24 & $\mathrm{E}$ & 4 \\
\hline \multicolumn{7}{|l|}{ MIMOSACEAE } \\
\hline Acacia piauhiensis Benth. & calumbi-branco & 1 & 4 & 0,24 & $\mathrm{~N}$ & 5 e 6 \\
\hline A. paniculata Willd. & unha-de-gato & 21 & 3 & 1.26 & $\mathrm{~N}$ & 5 \\
\hline Anadenanthera colubrina (Vell.) Brenan var. cebil & angico & 2 & 8 & 0,48 & $\mathrm{~N}$ & 3 e 6 \\
\hline Leucaena leucocephala (Lam.) De Wit. & leucena & 1 & 4 & 0,24 & $\mathrm{E}$ & $2,4 \mathrm{e} 6$ \\
\hline Piptadenia stipulacea (Benth.) Ducke & calumbi & 6 & 24 & 1,44 & $\mathrm{~N}$ & 5 e 6 \\
\hline Prosopis juliflora (Sw.) DC. & algaroba & 10 & 40 & 2,39 & $\mathrm{E}$ & 2 e 6 \\
\hline \multicolumn{7}{|l|}{ MORACEAE } \\
\hline Artocarpus integrifolia L. & jaca & 3 & 12 & 0,72 & $\mathrm{E}$ & 1 e 6 \\
\hline \multicolumn{7}{|l|}{ MUSACEAE } \\
\hline Musa paradisiaca $\mathrm{L}$. & banana & 15 & 60 & 3,59 & $\mathrm{E}$ & 1 e 3 \\
\hline Musa sp. 1 & banana-baé & 1 & 4 & 0,24 & $\mathrm{E}$ & 1 \\
\hline Musa sp. 2 & banana-pão & 1 & 4 & 0,24 & $\mathrm{E}$ & 1 \\
\hline \multicolumn{7}{|l|}{ MYRTACEAE } \\
\hline Eucalyptus globulus Labill. & eucalipto & 2 & 8 & 0,48 & $\mathrm{E}$ & 3 \\
\hline Eugenia uniflora L. & pitanga & 2 & 8 & 0,48 & $\mathrm{E}$ & 1 e 3 \\
\hline Myrciaria cauliflora (Mart.) O. Berg & jaboticaba & 2 & 8 & 0,48 & $\mathrm{~N}$ & 1 \\
\hline Psidium guajava $\mathrm{L}$. & goiaba & 17 & 68 & 4,07 & $\mathrm{E}$ & 1,3 e 6 \\
\hline Syzygium jambos L. & jambo-rosa & 1 & 4 & 0,24 & $\mathrm{E}$ & 1 e 6 \\
\hline Eugenia malaccensis L. & jambo & 2 & 8 & 0,48 & $\mathrm{E}$ & 1 e 6 \\
\hline \multicolumn{7}{|l|}{ NYCTAGINACEAE } \\
\hline Bougainvillea spectabilis Willd. & buguenvilha & 1 & 4 & 0,24 & $\mathrm{E}$ & 4 \\
\hline Guapira laxa (Netto) Furlan & piranha & 3 & 12 & 0,72 & $\mathrm{~N}$ & 4 \\
\hline \multicolumn{7}{|l|}{ OLEACEAE } \\
\hline Indeterminada 1 & azeitona & 4 & 16 & 0,96 & $\mathrm{E}$ & 1 e 6 \\
\hline \multicolumn{7}{|l|}{ FABACEAE } \\
\hline Cajanus indicus Spreng & feijão-guandu & 1 & 4 & 0,24 & $\mathrm{E}$ & 1 e 7 \\
\hline Indeterminada 2 & & 1 & 4 & 0,24 & $\mathrm{~N}$ & 5 \\
\hline Indeterminada 3 & rabo-de-cavalo & 1 & 4 & 0,24 & $\mathrm{~N}$ & 5 \\
\hline \multicolumn{7}{|l|}{ PLUMBAGINACEAE } \\
\hline Plumbago sp. & nuvem & 1 & 4 & 0,24 & $\mathrm{E}$ & 4 \\
\hline \multicolumn{7}{|l|}{ PUNICACEAE } \\
\hline Punica granatum L. & romã & 5 & 20 & 1,20 & $\mathrm{E}$ & 3 e 4 \\
\hline \multicolumn{7}{|l|}{ RHAMNACEAE } \\
\hline Ziziphus joazeiro Mart. & juá & 2 & 8 & 0,48 & $\mathrm{~N}$ & 3 e 6 \\
\hline \multicolumn{7}{|l|}{ ROSACEAE } \\
\hline Rosa sp. & rosa & 5 & 20 & 1,20 & $\mathrm{E}$ & 4 \\
\hline \multicolumn{7}{|l|}{ RUTACEAE } \\
\hline Citrus aurantium L. & laranja & 3 & 12 & 0,72 & $\mathrm{E}$ & 1 e 3 \\
\hline C. limonum Risso & limão & 16 & 64 & 3,83 & $\mathrm{E}$ & 1 e 3 \\
\hline Citrus sp. & laranja-cravo & 3 & 12 & 0,72 & $\mathrm{E}$ & 1 \\
\hline Ruta graveolens L. & arruda & 1 & 4 & 0,24 & $\mathrm{E}$ & 3 \\
\hline Murraya paniculata (L.) Jacq. & jasmim & 2 & 8 & 0,48 & $\mathrm{E}$ & 4 \\
\hline \multicolumn{7}{|l|}{ SAPINDACEAE } \\
\hline Talisia esculenta Radlik. & pitomba & 4 & 16 & 0,96 & $\mathrm{E}$ & 1 e 6 \\
\hline
\end{tabular}


Tabela 1 (continuação)

\begin{tabular}{|c|c|c|c|c|c|c|}
\hline Família/Espécie & Nome vulgar & Oc. & $\mathrm{Ab}$. & Fr. & Status & Usos \\
\hline \multicolumn{7}{|l|}{ SOLANACEAE } \\
\hline Solanum stipulaceum Roem. e Schult. & mela-bode & 1 & 4 & 0,24 & $\mathrm{~N}$ & 3 \\
\hline Solanum sp. & pimentinha & 1 & 4 & 0,24 & $\mathrm{~N}$ & 7 \\
\hline Nicotiana glauca Grah. & apara-raio & 1 & 4 & 0,24 & $\mathrm{~N}$ & 7 \\
\hline \multicolumn{7}{|l|}{ VERBENACEAE } \\
\hline Lippia alba (Mill.) N.E. Br. & erva cidreira & 1 & 4 & 0,24 & $\mathrm{E}$ & 3 \\
\hline \multicolumn{7}{|l|}{ VITACEAE } \\
\hline Leea sp. & café & 1 & 4 & 0,24 & $\mathrm{E}$ & 4 \\
\hline
\end{tabular}

Kumar et al. 1994). Quanto à estrutura, mostraram-se complexos e multiestratificados, com tamanho e formatos variados, mas na sua maioria retangulares, variando de 140 a $12.500 \mathrm{~m}^{2}$, com tamanho médio de $3.300 \mathrm{~m}^{2}$ e desvio padrão de $4.210 \mathrm{~m}^{2}$. A idade dos quintais variou entre 3 e 58 anos (com média de 25 anos), não havendo correlação com a riqueza ou abundância $(p>0,05)$, verificando-se o mesmo no que se refere à idade dos mantenedores $(p>0,05)$. Tais dados não corroboram com os descritos por Clerck \& Negrero-Castillo (2000) no México, bem como por Kehlenbeck \& Mass (2004) e Coomes \& Ban (2004), para Indonésia e Peru, respectivamente, e por WinklerPrins (2002) para os quintais do Pará, onde concluem que os quintais mais velhos tendem a ser mais diversos e estruturalmente mais complexos.

A estrutura vertical segue o padrão típico de quintais tropicais que apresentam em média três a quatro estratos, podendo chegar até seis em alguns quintais de ambiente úmidos (Nair 2004). Em Riachão de Malhada de Pedra, padrão semelhante foi registrado, apresentando três níveis: A) predominantemente baixo $(1 \geq 3 \mathrm{~m})$, constituído, principalmente, por espécies frutíferas e medicinais, como Anacardium occidentale, Malpighia glabra, Spondias purpurea, S. mombim, Psidium guajava, Plectranthus sp., Comiphora leptophloeos e Citrus limonum; B) médio $(3 \geq 7 \mathrm{~m})$, englobando as frutíferas e as plantas de sombra, como Prosopis juliflora, Anonna squamosa, A. occidentale, Cordia trichotoma e Musa paradisiaca; C) alto ( $7 \geq 12 \mathrm{~m})$, com Cocos nucifera, Prosopis juliflora, Artocarpus integrifolia, Anadenanthera colubrina, Crataeva tapia, Schinopsis brasiliensis, A. occidentale, Ziziphus joazeiro, Eugenia uniflora, Attalea geraensis (Fig. 1).

A localização das espécies nos quintais tem como referência a unidade residencial. As espécies ornamentais estão sempre em frente à propriedade ou circundado-a, o que obviamente reflete o interesse pela estética do quintal, o que tem sido evidenciado em muitos trabalhos (Lamont et al. 1999; Murrieta \& WinklerPrins 2003; Wezel \& Bender 2003). Segundo Lamont et al. (1999) a presença dessas espécies está associada à proximidade dos centros urbanos, mas há um significado cultural e estético muito evidente na sua seleção (Murrieta \& WinklerPrins 2003). Nas áreas destinadas à monocultura do milho (Zea mays L.), feijão (Phaseolus vulgaris L.), palma forrageira (Opuntia sp.) e macaxeira (Manihot sp.), encontram-se associações com outras espécies, como mamão $(C$. papaia), parecendo refletir um padrão comum para os quintais de semi-árido (Wezel \& Bender 2003; Albuquerque et al. 2005).

As plantas medicinais e condimentares de porte herbáceo são cultivadas em áreas restritas onde recebem maiores cuidados, geralmente próximo à cozinha, pois facilita os cuidados, uma vez que a mulher é a principal responsável por essas espécies. Tal situação é comum em quintais de outras regiões (Lamont et al. 1999; Murrieta \& WinklerPrins 2003; Wezel \& Bender 2003).

Usos - Nos quintais estudados foram observadas espécies com múltiplos usos, como medicinais, ornamentais e madeireiras. Sobre esta última categoria, Shavanas \& Kumar (2003) encontraram nos quintais de Kerala (Índia) uma grande diversidade de plantas nativas e exóticas cultivadas para obtenção de combustível, espécies selecionadas pela população local pelo potencial energético das mesmas. Para o autor, os quintais podem ser áreas de cultivo contínuo de espécies para este fim, constituindo em uma saída para redução no impacto ambiental, podendo contribuir para conservação da diversidade local.

Os quintais são áreas com recursos contínuos e possuem várias funções, sendo a principal, em todas as regiões, a produção de alimentos como mostra 
Wezel \& Bender (2003) para os quintais de Cuba, os quais constituem um importante fator para economia local e a auto-suficiência para muitas famílias.

As plantas dos quintais estudados são usadas para diversos fins, refletindo a mesma situação apontada em outros estudos (Nair 1986; Lamont et al. 1999; Wezel \& Bender 2003; Albuquerque et al. 2005).
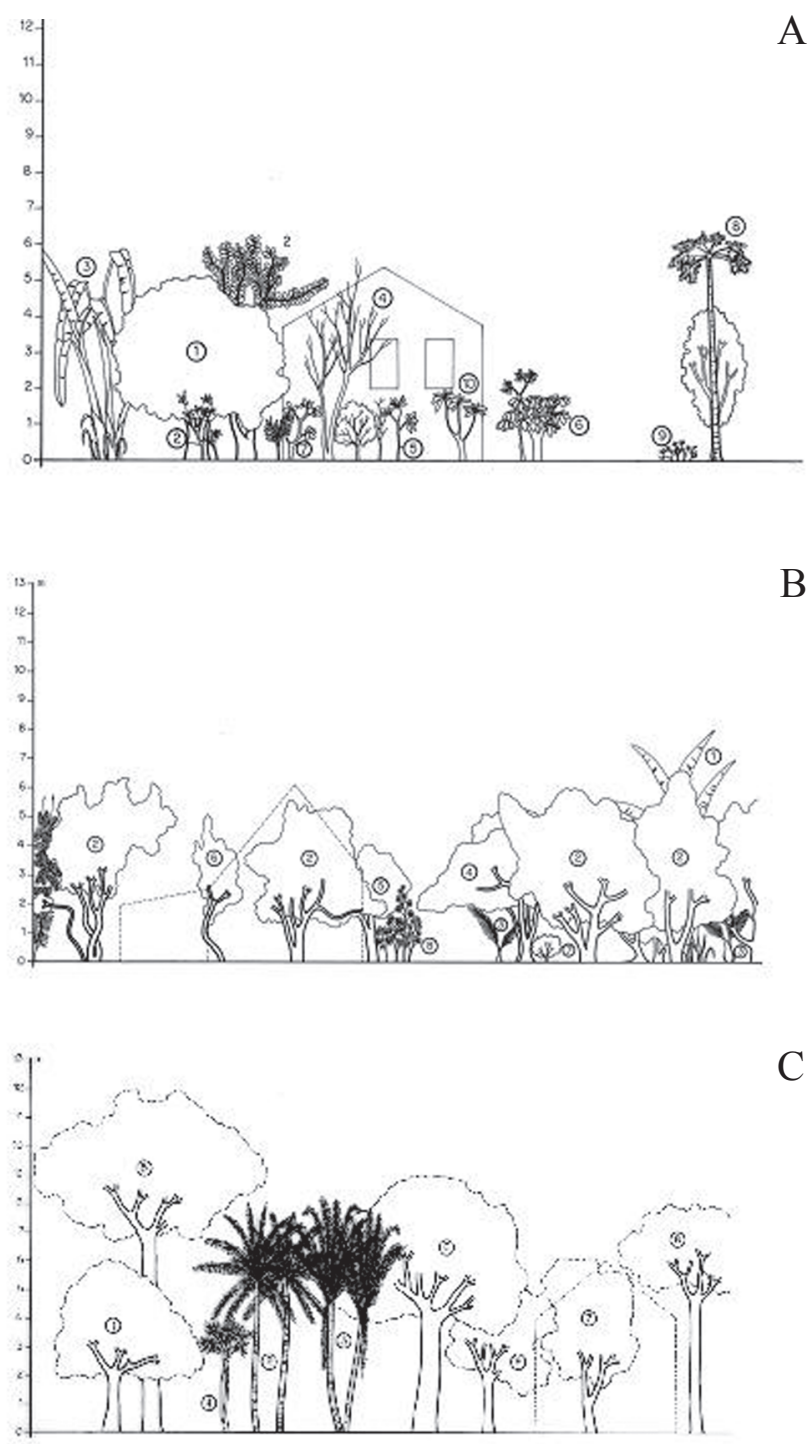

$\mathrm{C}$

Figura 1. Perfil da estrutura vertical dos três tipos predominantes de quintais agroflorestais da comunidade de Riachão de Malhada de Pedra, município de Caruaru, Pernambuco (Nordeste do Brasil), representada por três níveis de estratificação: A. 1. Mangifera indica; 2. Psidium guajava; 3. Musa paradisiaca; 4. Annona squamosa; 5. Leea sp. ; 6. Schefflera arboricola; 7. Hibiscus rosa-sinensis; 8. Carica papaya; 9. Geranium sp.; 10. Nerium oleander. B. 1. Musa paradisiaca; 2. Anacardium occidentale; 3. Cocos nucifera; 4. Comiphora leptophloeos; 5. Artocarpus integrifolia; 6. Annona muricata; 7. Citrus aurantium; 8. Spondias purpurea. C. 1. Talisia esculenta; 2. Cocos nucifera; 3. Syagrus sp.; 4. Psidium guajava; 5. sp. 1; 6. Ziziphus juazeiro; 7. Erythrina velutina; 8. Schinopsis brasiliensis.
Contudo, a finalidade principal dos quintais de Riachão de Malhada de Pedra é de promover a complementação alimentar das unidades familiares, com exceção apenas de um quintal, cujo proprietário vende o excesso dos frutos produzidos na feira livre de Caruaru, principalmente de Anacardium occidentale (espécie predominante no quintal). As espécies alimentícias são representadas por frutíferas, tubérculos e condimentos, mas apenas as frutíferas (lenhosas) se mantêm no quintal de forma contínua. Algumas espécies, como C. papaya, A. occidentale e $M$. paradisiaca, possuem alta freqüência nos quintais de Riachão de Malhada de Pedra, devido a sua grande importância na complementação alimentar da população local.

Apesar de todas as espécies serem consideradas úteis, observa-se que apenas um pequeno número é utilizado para mais de uma finalidade, o que não corrobora os dados de outros autores como, por exemplo, Angel-Perez \& Martin (2004) que trabalhando com quintais de Veracruz, no México, encontraram uma alta diversidade de espécies de múltiplos usos. Para algumas espécies, são atribuídos mais de um tipo de uso, como ocorre com as alimentícias também usadas como medicinais, a exemplo: Anacardium occidentale, Eugenia uniflora, Musa paradisiaca, Citrus aurantium e C. limonum, estas duas últimas empregadas como antigripais em adultos e crianças e também para curar doenças comuns em galinhas. Entre as espécies mais versáteis, pode-se destacar Senna martiana usada como forragem, ornamental e sombra.

O maior percentual de uso foi registrado para plantas alimentícias $(28,83 \%)$, seguidas de ornamental $(27,03 \%)$, medicinal $(14,42 \%)$, sombra (geralmente plantas de cobertura ampla fechada que proporcionam sombreamento natural) $(12,62 \%)$, madeireira $(8,11 \%)$, forragem $(2,7 \%)$ e outros usos $(6,31 \%)$. Nesta última categoria incluem-se os usos místicos e tecnológicos. A proporção entre espécies nativas e exóticas, por categorias de uso, é significativamente maior para as espécies nativas nas categorias madeireira e forragem (Teste- $\mathrm{G}=14,96 ; \mathrm{p}<0,05$ ).

$\mathrm{Na}$ área de estudo, algumas plantas lenhosas são mantidas na propriedade exclusivamente para produção de lenha, como Acacia paniculata, Piptadenia stipulacea e Acacia piauhiensis. Outra planta, Nicotiana glauca, é usada pelos mantenedores como proteção contra raios em períodos chuvosos. $\mathrm{Na}$ categoria de uso tecnológico, Capparis hastata é utilizada na produção de artigos artesanais como chaveiros e adornos que são comercializados na feira 
de Caruaru. Na mesma categoria, destaca-se Cordia trichotoma, manejada nos quintais para o fabrico de ferramentas de uso doméstico. A madeira dessa espécie é considerada muito resistente e flexível, por isso possui a preferência dos mantenedores para a confecção de cabos de enxada.

Muitas plantas exóticas são preferencialmente cultivadas nos quintais de Riachão de Malhada de Pedra, sobretudo às espécies ornamentais, como algumas Asteraceae e Rosaceae, e frutíferas como Anacardium occidentale, Musa paradisiaca, Carica papaya e Citrus limonum, semelhante ao encontrado por outros autores para quintais de regiões úmidas e áridas (Rico-Gray et al. 1990; Lamont et al. 1999; Wezel \& Bender 2003; Albuquerque et al. 2005).

Uma planta abundante nos quintais foi o caju (A. occidentale), consumida de várias formas, desde in natura ao uso da castanha torrada e pisada no pilão, servida como farinha, sendo um complemento dietético preferencial, principalmente para as crianças, devido ao sabor agradável. Além das frutíferas, são cultivadas outras espécies alimentícias como, tomate, plantas condimentares (pimenta), cereais e tubérculos. Padrão semelhante foi encontrado nos quintais de Alagoinha, Nordeste do Brasil, onde Albuquerque et al. (2005) registraram uma alta densidade de espécies frutíferas, muitas delas comuns às de Riachão de Malhada de Pedra, como manga, laranja, goiaba, caju e mamão.

Manejo - O manejo nessas práticas é simples e de baixo custo, uma vez que envolve práticas tradicionais de cultivo de plantas (Albuquerque 2005). Nos quintais estudados, o manejo envolve tecnologias simples, ficando os cuidados mais intensos para as espécies que garantem alimento à família, como Zea mays, Manihot sp. e Phaseolus sp., além de algumas frutíferas. No caso das frutíferas, destaca-se M. paradisiaca que recebe cuidados especiais de manejo, principalmente em períodos secos, quando são adubadas com esterco de gado e irrigadas diariamente. Em outras ocasiões, a espécie é plantada em áreas mais sombreadas, como ao lado da casa ou próximo a arbóreas de copa ampla.

A demanda de produtos nos quintais é maior nos períodos chuvosos, devido à facilidade na obtenção de água para irrigar as plantações e à ação direta das chuvas. Neste período outras espécies são cultivadas, como (Cucumis sp.) maxixe, Abelmonchus sp. (quiabo), Citrullus sp. (melancia) e Curcubita sp. (abóbora) associadas às plantações pré-existentes. Nos anos mais secos, algumas espécies frutíferas diminuem a produção e a qualidade dos frutos como é o caso de Psidium guajava e Annona squamosa.

A preferência pelo cultivo de plantas lenhosas segue um padrão para todos os quintais, o que pode ser justificado principalmente devido à resistência destas espécies às adversidades climáticas, sendo mantidas sem muitas exigências de manejo. Além disso, as arbóreas favorecem o estabelecimento de um microclima favorável e ao desenvolvimento de outras espécies (Wezel \& Bender 2003; Nair 2004).

Segundo WinklerPrins (2002) cerca de $70 \%$ dos quintais do Pará são mantidos sob a responsabilidade da mulher. Avaliando-se as práticas de manejo isoladamente, observou-se que há uma distribuição das atividades, ou seja, nas áreas de monocultura os cuidados são de responsabilidade dos homens, restando para as esposas e os filhos os cuidados com as espécies frutíferas, ornamentais e condimentares. O papel das mulheres nos quintais é fundamental na manutenção e na decisão das espécies a serem cultivadas, sobretudo das ornamentais e medicinais. Em alguns casos as mulheres são as mantenedoras diretas das práticas $(12,5 \%)$.

Origem das espécies nos quintais - As espécies nos quintais estudados são obtidas por meio de parentes, amigos ou vizinhos ( $86 \%$ ) e, raramente, são compradas $(16 \%)$. No caso das ornamentais, muitas são oriundas de outras localidades, geralmente trazidas por um parente. As plantas que surgem espontaneamente ou através de rebrota são mantidas, na maioria dos casos $(87,5 \%)$ por possuírem alguma finalidade. E o caso dos quintais de Cuba, onde as medicinais existentes nos quintais são, em sua maioria, espontâneas (Wezel $\&$ Bender 2003). Na pesquisa, a espécie mais freqüente nos quintais é a vassourinha de botão (Borreria verticillata Mayer), usada como sugere o próprio nome, na confecção de vassoura.

Apenas uma minoria (12,5\%) dos mantenedores elimina as plantas por conveniência e estética; em um caso isolado, indivíduos da mesma espécie são eliminados para evitar a competição intraespecífica, como é o caso de $A$. occidentale, refletindo o conhecimento diante de um processo ecológico comum.

Em conclusão, observou-se que em Riachão de Malhada de Pedra, a diversidade e a composição das espécies são mais influenciadas por aspectos culturais e nutricionais do que por fatores externos (como a proximidade com a vegetação nativa). As espécies nativas apresentaram-se como um importante 
componente nos quintais, podendo contribuir para o uso sustentável dos recursos naturais da caatinga, indicando que um plano de manejo para estas áreas poderia reduzir a pressão sobre a vegetação local.

\section{Agradecimentos}

À Empresa Pernambucana de Pesquisa Agropecuária (IPA), unidade Caruaru, pelo apoio logístico; à Coordenação de Aperfeiçoamento de Pessoal de Nível Superior (CAPES), pela bolsa concedida à primeira autora; ao $\mathrm{CNPq}$, pelo apoio financeiro dado a Ulysses P. Albuquerque; ao Laboratório de Etnobotânica Aplicada, da Universidade Federal Rural de Pernambuco, pelo apoio e subsidio na pesquisa; aos informantes da comunidade de Riachão de Malhada de Pedra, pelo inestimável apoio e permissão para estudar os seus quintais; à Dra. Laise de H. Cavalcanti Andrade, pela leitura crítica deste trabalho; aos revisores anônimos, pelas sugetões.

\section{Referências bibliográficas}

Albuquerque, U.P. 2005. Etnobiologia e Biodiversidade. Recife, NUPEEA/Sociedade Brasileira de Etnobiologia e Etnoecologia.

Albuquerque, U.P. \& Lucena, R.F.P. 2004. Métodos e técnicas para a coleta de dados. Pp. 37-62. In: U.P. Albuquerque \& R.F.P. Lucena (orgs.). Métodos e técnicas na pesquisa etnobotânica. Recife, Editora Livro Rápido/NUPEEA.

Albuquerque, U.P.; Cavalcanti, L.H. \& Caballero, J. 2005. Structure and Floristics of Homegardens in Northeastern Brazil. Journal of Arid Enviroments 62(3): 491-506.

Albuquerque, U.P.; Lucena, R.F.P.; Monteiro, J.M.; Florentino, A.T.N. \& Almeida, C.F.C.B.R. 2006. Evaluating two quantitative ethnobotanical techniques. Ethnobotany Research \& Applications 4(1): 51-60.

Anderson, A.B.; Gely, A.; Strudwick, J.; Sobel, G.L. \& Pinto, M.G.C. 1985. Um sistema agroflorestal na várzea do estuário amazônico (Ilha das Onças, Município de Barcarena, Estado do Pará). Acta Amazônica 15: 195-224.

Andrade-Lima, D. 1981. The Caatingas Dominium. Revista Brasileira de Botânica 4: 149-153.

Angel-Perez, A.L.D. \& Martin, A.M.B. 2004. Totonac homegardens and natural resources in Veracruz, México. Agriculture and Human Values 21: 329-346.

Araújo, E.L. \& Ferraz, E.M.N. 2004. Amostragem da vegetação e índices de diversidade. Pp. 89-137. In: U.P. Albuquerque \& R.F.P. Lucena (orgs.). Métodos e técnicas na pesquisa etnobotânica. Recife, Editora Livro Rápido/NUPEEA.

Ayres, M.; Ayres, M.J.; Ayres, D.L. \& Santos, S.A. 2000. Bioestat 2.0: aplicações estatísticas nas áreas das ciências biológicas e médicas. Brasília, Sociedade Civil Mamirauá/CNPq.
Barrera, A. 1980. Sobre la unidad de habitación tradicional campesina y el manejo de recursos bióticos en el area Maya Yucatense. Biotica 5: 115-129.

Beatriz, R.A.; Graciela, G.S.; Isabel, O.D.M.; Virgínia, O.C.; Elvira, P.P.R.; Estela, P.G. \& Lídia, R.H. 1998. Theobroma bicolor Humb. \& Bonpl. (Sterculiaceae, "cuapataxtle") en el Municipio de Ayutla de Los Libres, Guerrero. Composición florística de los huertos y aspectos etnobotánicos. Boletim da Sociedade Botánica de México 63: 75-84.

Blanckaert, I.; Swennen, L.R.; Flores, M.P.; Lopez, R.R. \& Saade, L. 2002. Floristic composition, plants uses and management pratices in homegardens of San Rafael, Coxcatlán, Valley of Tehuacatlán, México. Journal of Arid Environments 57: 39-62.

Caruaru - O portal. Apresenta informações sobre o município de Caruaru - Disponível em http:// www.caruaru.org/geografia.htm (Acesso em: 5/06/2005).

Clerck, E.A.L. \& Negrero-Castillo, P. 2000. P. Plant species of traditional Mayan homegardens of Mexico an analogs for multistrata agroforests. Agroforestry Systems 48: 303-317.

Coomes, O.T. \& Ban, N. 2004. Cultivated plants species diversity in homegardens of an Amazonian peasant village in northeastern Peru. Economic Botany 58(3): 420-434.

Diegues, A.C. \& Arruda, R.S.V. 2001. Saberes tradicionais e biodiversidade no Brasil. Brasília, Ministério do Meio Ambiente; São Paulo, USP. (Biodiversidade, 4).

Emperaire, L. \& Pinton, F. 1986. Dona Flora et les cajous: deux systèmes agricoles au sud-est du Piauí (Brèsil). Journal d'Agriculture Traditionel et de Botanique Appliqué 33: 193-212.

Fernandes, E.C.M. \& Nair, P.K.P. 1986. An evaluation of the structure and function of tropical homegardens. Agricultural Systems 21: 279-310.

FIDEM - Fundação de Desenvolvimento Municipal. Desenvolvido pela Secretaria de Planejamento e Desenvolvimento Social. Apresenta informações sobre os Perfis Municipais. Disponível em http:// www.fidem.pe.gov.br (Acesso em: 10/05/2005).

Kehlenbeck, K. \& Mass, B.L. 2004. Crop diversity and classification of homegardens in Central Sulawesi Indonésia. Agroforestry Systems 63: 53-62.

Kumar, B.M.; George, S.J.; Chinnamani, S. 1994. Diversity, struture and standing stock of wood in tge homegardens of Kerala in Peninsular India. Agroforestry Systems 25: 243-262.

Lamont, S.R.; Eshbaugh, W.A. \& Greenberg, A.M. 1999. Composition, diversity, and use of homegardens among three Amazonian villages. Economic Botany 53(3): 312-326.

Lucena, RF.P.; Albuquerque, U.P.; Monteiro, J.M.; Almeida, C.F.C.B.R.; Florentino, A.T.N. \& Ferraz, J.S.F. 2007. Useful plants of the semi-arid northeastern region of Brazil - a look at their conservation and sustainable use. Environmental Monitoring and Assessment 125: 281-290. 
Maia, G.N. 2004. Caatinga: árvores e arbustos e suas utilidades. São Paulo, D \& Z Computação Gráfica e Editora.

Millat-e-Mustafa, M.D. 1998. An approach towards analysis of homegardens. Pp. 39-48. In: A. Rastogi; A. Godble \& P. Shengji (eds.). Applied Ethnobotany in natural resource management traditional home gardens. Nepal, International Centre for Integrated Mountain Development Kathmandu.

MMA - Ministério do Meio Ambiente. 2002. Avaliação e ações prioritárias para a conservação da biodiversidade da Caatinga. Universidade Federal de Pernambuco/ Fundação de Apoio ao desenvolvimento da conservação do Brasil. Fundação Biodiversidade. Brasília, Embrapa Semi-árido.

Monteiro, J.M.; Albuquerque, U.P.; Lins Neto, E.M.F.; Araujo, E.L. \& Amorim, E.L.C. 2006a. Use patterns and knowledge of medicinal species among two rural communities in Brazil's semi-arid northeastern region. Journal of Ethnopharmacology 105: 173-186.

Monteiro, J.M.; Almeida, C.F.C.B.R.; Albuquerque, U.P.; Florentino, A.T.N. \& Oliveira, R.L.C. 2006b. Use and traditional management of Anadenanthera colubrina (Vell.) Brenan in the semi-arid region of northeastern Brazil. Journal of Ethnobiology and Ethnomedicine 2: 6.

Murrieta, R.S.S. \& WinklerPrins, A.M.G.A. 2003. Flowers of water: homegardens and gender roles in a riverine caboclo community in the lower Amazon, Brazil. Culture and Agriculture 25: 35-47.

Nair, P K.P. 1986. An Evaluation of the Struture and Function of Tropical Homegardens. Agricultural Systems 21: 279-310.

Nair, P.K.P. 1993a. An introduction to Agroforestry. ICRAF/ Kluwer Academic Publishers.

Nair, P.K.P. 1993b. State-of-the-art of agroforestry systems. Forest Ecology and Management 45: 5-29

Nair, P.K.P. 2001. Do tropical homegardens elucidate science, or is it the other way around? Agroforestry Systems 53: 239-245.

Nair, P.K.P. 2004. The enigma of tropical homengardens. Agroflorestry Systems 61: 135-152.

Padoch, C. \& Jong, W. 1991. The House Gardens of Santa Rosa: diversity and variability in an Amazonian Agricultural System. Economic Botany 45(2): 166-175.
Rico-Gray, V.; Gomez-Pompa, A. \& Chan, C. 1985. Las selvas manejadas por los Mayas de Yohaltun, Campeche, México. Biotica 10(4): 321-327.

Rico-Gray, V.; Garcia-Franco, J.G.; Chemas, A.; Puch, A. \& Sima, P. 1990. Species composition, similarity, and structure of Mayan Homegardens in Tixpeual and Tixcacaltuyub, Yucatan, Mexico. Economic Botany 44: 470-487.

Rugalema, G.H.; Johnsen, F.H.; Okting'ati, A. \& Minjas, A. 1994. The homegarden agroforest system to Bukoba district, North-Western Tanzania. 3. An economic appraisal of possible solutions to falling productivity. Agroforestry Systems 28: 227-236.

Sampaio, E.V.S.B. 2002. Uso das plantas da Caatinga. Pp. 49-90. In: E.V.S.B. Sampaio; A.M. Giulietti; J. Virgínio \& C.F.L. Gamarra-Rojas (orgs.). Vegetação e flora da Caatinga. Recife, APNE /CNIP.

Santos, S.R.M.; Miranda, I.S. \& Tourinho, M.M. 2004. Análise florística e estrutural de sistemas agroflorstais das várzeas do rio Juba, Cametá, Pará. Acta Amazônica 34(2): 251-263.

Shavanas, A. \& Kumar, B.M. 2003. Fuelwood characteristics of tree species in the homegardens of Kerala, Índia. Agroforestry Systems 58: 11-24.

Soemarwoto, O.; Soekartadiredja, E.M.E. \& Ralam, A. 1985. The Javanese home-garden as an integrated agroecossystem. Food and Nutrition Bulletin 7(3): 44-47.

Sokal, R.R. \& Rolf, F.J. 1995. Biometry. New York, W.H. Freeman and Company.

Torquebiau, E. 1992. Are tropical agroforestry homegardens sustainable? Agriculture, Ecossystems and Environment 41: 189-207.

Wezel, A. \& Bender, S. 2003. Plant species diversity of homegardens of Cuba and its significance for household food supply. Agroforestry Systems 57: 39-49.

Wiersum, K.F. 2004. Forest gardens as an 'intermediate' landuse system in the nature-culture continuum: characteristics and future potential. Agroforestry Systems 61: 123-134.

WinklerPrins, A.M.G.A. 2002. House-lot gardens in Santarém, Pará, Brazil: Linking rural with urban. Urban Ecosystems 6: 43-65. 